\title{
Evaluation of Sodium Bicarbonate as an Adjunct to Non Surgical Periodontal Therapy and its Effect on Oxidative Stress: A Clinico-biochemical Study
}

\author{
Shaila Kothiwale*, Meenal Kella, Laxmi Hombal and Amrita Rathore \\ Department of Periodontics, KLE VK Institute of Dental Sciences, KLE University, Belgaum, India
}

Received: June 24,2014; Accepted: June 30,2014; Published: July 02,2014

*Corresponding author: Shaila V. Kothiwale, Professor, Department of Periodontics, KLE VK Institute of Dental Sciences, Belgaum. Phone: 9448822706 , E-mail: shailakothiwale2000@yahoo.co.in

\begin{abstract}
Objectives: The purpose of the interventional study was to determine the effects of sodium bicarbonate as an adjunct to non surgical periodontal therapy in chronic periodontitis by measuring salivary malondiadehyde (MDA) levels.

Methods: The study included 30 chronic periodontitis patients who were randomly assigned to test and control group. Patients were subjected to scaling and root planing. In test group, subjects were instructed to massage their teeth and gingiva with sodium bicarbonate slurry twice daily after regular brushing. Clinical parameters of Gingival Index, Plaque Index, Probing Pocket Depth and Clinical attachment level and whole saliva samples were obtained from both groups at baseline and 3 months. The salivary MDA levels were measured using colorimetric assay procedure. The Student $\mathrm{t}$ test was used to analyze all parameters.
\end{abstract}

Results: At 3 months follow up all the clinical parameters in both the group were significantly reduced $(\mathrm{p}<0.001)$. The gingival index score showed statistically significant reduction in test group $(1.2 \pm 4.8)$ and control group $(0.28 \pm 0.18)$ at 3 month interval. The difference in MDA levels at baseline and 3 months was $(4.8 \pm 1.94)$ in test group and $(1.8 \pm 0.81)$ in control group. The magnitude of difference in gingival index and MDA levels in the test group was greater on intergroup comparison.

Conclusions: The present study showed positive outcome in all the clinical parameters and significant reduction in salivary MDA levels with the adjunct use of sodium bicarbonate to non-surgical periodontal therapy.

Keywords: Sodium bicarbonate; Gingival massage; Nonsurgical therapy; Periodontitis; Malondiadehyde

\section{Introduction}

Periodontits is a chronic inflammatory disease of supporting tissues of the teeth resulting in progressive destruction of the periodontal ligament and alveolar bone.Patients with periodontal disease are more susceptible to an imbalance of antioxidant-oxidative stress situation. Reactive oxygen species released during oxidative stress can be measured by the final product of LPO, such as MDA which is the principal and most studied product of polyunsaturated fatty acid peroxidation. As whole saliva constitute a first line of defence against free radical mediated oxidative stress. MDA that is realeased during oxidative stress becomes one constituent of saliva and can be used as a biomarker to asses the inflamatory condition as sampling of saliva is easy, non invasive and it represents pooled sample with contribution from all periodontal sites. Thus salivary MDA levels were used to detect the inflammatory state in the study.

Bacterial plaque is generally considered to be the main etiological agent. When stimulated by bacterial pathogens, host cells (eg., PMNs) release reactive oxygen species (ROS) as part of the immune response. The interactions between pathogen and host defensive capacity result in periodontal tissue breakdown. Oxidative stress lies at the heart of this periodontal tissue damage as a result of host-microbial interactions ${ }^{[1]}$.

Hence, periodontal therapy has been directed at altering the periodontal environment to one which is less conducive to the retention of bacterial plaque in the vicinity of gingival tissue. It comprises of non-surgical therapy and surgical therapy. Current therapeutic guidelines for chronic periodontitis emphasize the need for control of bacterial population by scaling and root planning and maintenance of plaque control by means of patient home care. In this form of disease, the inability to control the clinical signs of disease results from either inadequate homecare or inadequate access for subgingival cleaning. Therefore, nonsurgical periodontal therapy remains the core component and mainstay of successful periodontal therapy ${ }^{[2]}$.

The use of a chemical agent as an adjunct to the non surgical therapy will greatly enhance patient's oral hygiene and motivation. Sodium biocarbonate $\left(\mathrm{NaHCO}_{2}\right.$, baking soda) is one such agent which has been widely used. ${ }^{[3]}$ The appeal of sodium biocarbonate products seems to lie in its safety, low cost, low abrasivity, water solubility, buffering ability, compatibility with fluoride, and in high concentrations, antimicrobial properties ${ }^{[4]}$.

Over the years, antimicrobial agents, such as sodium bicarbonate, have been incorporated into mouthrinses and dentrifices in an attempt at providing a practical approach toward 
improving oral health. Moreover, bicarbonate in saliva buffers \& neutralizes plaque acids. Sodium bicarbonate tends to maintain a $\mathrm{pH}$ of 8.1 when acids, which lower $\mathrm{pH}$, or bases, which raise $\mathrm{pH}$, are added to the solution. In other words, sodium bicarbonate should raise salivary $\mathrm{pH}$ that is lower than 8 (i.e. increase saliva alkalinity). Additionally, sodium bicarbonate due to its slightly abrasive consistency works as a mechanical cleanser of teeth and gingival tissues. ${ }^{[5]}$

A close relationship exists between plaque and chronic inflammatory periodontal disease and the effectiveness of conservative treatments used to reduce the severity of disease, is widely accepted. The study was carried out to assess the role of sodium bicarbonate, applied as an adjunct, after an exquisite full mouth scaling and root planning and to measure the levels of salivary MDA to quantify the levels of oxidative stress. Thus, the aim of this study was to determine the effects of sodium bicarbonate on the clinical parameters of periodontal disease and measure the levels of salivary MDA.

\section{Materials and methods}

The study was randomized, controlled, double blinded interventional trial which included 30 patients, both males and females, with age ranging from 25-55 years who reported to the Department of Periodontics. The research project was ethically approved by the Institutional Review board. The study was conducted in accordance with the World Medical Association Declaration of Helsinki and after obtaining informed consent from the participants.

The subjects with generalized chronic periodontitis (moderate) according to the 1999 AAP classification were included in the study. The sample size was determined based on the statistical formula. The subjects were randomly allocated to one of the two groups using a computer generated numbers and allocation was concealed until interventions were assigned for the interventional trial (Figure 1).

Subjects included in the study had at least 20 natural teeth present. The exclusion criteria were subjects with a history of smoking and/or alcoholism or tobacco chewers, use of mouthwashes, any systemic diseases/illness, pregnant women, subjects who had received antioxidant vitamins, antiinflammatory drugs, antibiotics, nutritional supplements or any other drugs over the past 6 months, those who had undergone any periodontal treatment over the past 6 months.

Following selection, clinical data included Gingival Index (GI), (Sillness and Loe 1963), Plaque Index (PI), Probing Pocket Depth (PPD) and Clinical attachment level (CAL). The data were assessed at the baseline and 3 months. Patients who participated in the study were refrained from eating and drinking for one hour and then asked to rinse their mouth with tap water. Then $2 \mathrm{ml}$ of unstimulated whole saliva was collected by spitting into sterile containers with subjects seated in an upright position. Collected samples were then transported to the laboratory for the measurement of MDA levels.

Full mouth scaling and root planning was performed by periodontist and all the clinical measurements were performed by the same examiner. The prematurities, caries, overhang were treated and home care oral hygiene instructions were given. In test group, subjects were asked to massage their teeth and gingiva with 1/2 teaspoon of Baking soda slurry (sodium Bicarbonate+ water) twice daily after routine brushing with toothpaste. Clinical parameters and salivary samples were collected at 3 months from both groups.

\section{Assay procedure}

In the present study, colorimetric assay procedure proposed by Okawa $\mathrm{H}$ et al (1979) ${ }^{[6]}$ and Satoh K (1978) ${ }^{[7]}$ was used. It is based on the reaction between thiobarbituric acid reactive substances (TBARS) and thiobarbituric acid (TBA) reagent to generate a pink colored product and determined at $535 \mathrm{~nm}$. The value obtained was then calculated against the standard curve and the results were estimated in $\mu$ mol MDA equivalents. Tetraethoxypropane was used as an external standard.

\section{Statistical analysis}

The Student t-test was used to analyse all parameters between study and control sites and to determine the significance of changes over time. 95\% of Confidence Interval (CI) was calculated. The significant level was fixed at 0.05 . SPSS version 17.0 was used to analyse the data.

\section{Results}

All the clinical parameters, GI, PI, PPD, CAL and salivary MDA levels measured at baseline and at 3 months interval. The gingival index score showed statistically significant reduction in test group $(1.2 \pm 4.8)$ and control group $(0.28 \pm 0.18)$ at 3 month interval. Post treatment scores showed statistically greater reduction in test group. On comparison of test and control groups statistically significant difference was observed $(\mathrm{p}<0.001)$ (Table 1). The plaque index score showed statistically significant reduction in test group (1.75 \pm 3.84$)$ and control group $(1.36 \pm 0.76)$ at 3 month interval. The intergroup comparison revealed statistically significant difference in plaque index score $(p<0.001)$.(Table 2$)$.

Table 1: Comparison of gingival index scores at baseline and three months interval between groups.

\begin{tabular}{|c|c|c|c|}
\hline \multicolumn{4}{|c|}{ Gingival index } \\
\hline Group & Day 0 & 3 months & Differences \\
\hline Test & $2.2 \pm .53$ & $0.98 \pm 0.46$ & $1.2 \pm 4.8$ \\
\hline Control & $2.5 \pm 2.1$ & $2.27 \pm 0.17$ & $0.28 \pm 0.18$ \\
\hline P value & 0.023 & $<0.001$ & $<0.001$ \\
\hline
\end{tabular}

Table 2: Comparison of plaque index scores at baseline and three months interval between groups.

\begin{tabular}{|c|c|c|c|}
\hline \multicolumn{4}{|c|}{ Plaque Index } \\
\hline Group & Day 0 & 3 months & Differences \\
\hline Test & $2.7 \pm 2.6$ & $0.95 \pm 0.25$ & $1.75 \pm 3.84$ \\
\hline Control & $2.8 \pm 2.2$ & $1.44 \pm 0.26$ & $1.36 \pm 0.76$ \\
\hline P & 0.298 & $<0.001$ & $<0.001$ \\
\hline
\end{tabular}


The difference between the CAL values at baseline and 3 months in test group was $0.85 \pm 0.42$ and in control group was $0.28 \pm$ 0.15 . The significant difference $(\mathrm{p}<0.001)$ in CAL values was observed when intergroup comparison was made. (Table3).The difference between the pocket probing depth values at baseline and 3 months in test group was $2.1 \pm 0.73$ and in control group was $1.4 \pm 0.73$. The intergroup comparison showed statistically significant difference $(\mathrm{p}<0.05)$.(Table 4$)$.

The difference in MDA levels at baseline and 3 months was $(4.8 \pm 1.94)$ in test group and $(1.8 \pm 0.81)$ in control group. Significantly lower MDA levels were seen in test group. The significant difference $(\mathrm{p}<0.001)$ was observed when intergroup comparison was made. (Table 5).Thus the results showed that the magnitude of difference observed in gingival index scores and MDA levels was greater when intergroup comparison was done.

\section{Discussion}

Periodontitis is a pathological condition in which chronic local inflammation exhibits an exacerbated inflammatory/immune response to the periodontopathogenic bacteria that accumulate around the tooth and gingival tissues which may in turn lead to tissue damage and may result in tooth loss. ${ }^{[8]}$ The reactive free radicals produced during chronic conditions like periodontitis may damage cells by initiation of lipid peroxidation that causes profound alteration in structural integrity and functions of cell membranes. They can be measured by levels of malondialdehyde. [9]

The present study was done to assess the effect of the

Table 3: Comparison of clinical attachment levels at baseline and three months interval between groups.

\begin{tabular}{|c|c|c|c|}
\hline \multicolumn{4}{|c|}{ Clinical Attachment Level } \\
\hline Group & Day 0 & 3 months & Differences \\
\hline Test & $3.5 \pm 0.28$ & $2.7 \pm 0.37$ & $0.85 \pm 0.42$ \\
\hline Control & $3.8 \pm 0.16$ & $3.5 \pm 0.18$ & $0.28 \pm 0.15$ \\
\hline P value & 0.005 & $<0.001$ & $<0.001$ \\
\hline
\end{tabular}

Table 4: Comparison of pocket probing depth scores at baseline and three months interval between groups.

\begin{tabular}{|c|c|c|c|}
\hline \multicolumn{4}{|c|}{ Pocket probing depth } \\
\hline Group & Day 0 & 3months & Differences \\
\hline Test & $7.2 \pm 1.2$ & $5.1 \pm 0.96$ & $2.1 \pm 0.73$ \\
\hline Control & $6.8 \pm 0.96$ & $5.4 \pm 0.99$ & $1.4 \pm 0.73$ \\
\hline P value & 0.509 & 0.201 & $<0.05$ \\
\hline
\end{tabular}

Table 5: Comparison of salivary MDA levels at baseline and three months interval between groups.

\begin{tabular}{|c|c|c|c|}
\hline \multicolumn{4}{|c|}{ Salivary MDA } \\
\hline Group & Day 0 & 3 months & Difference \\
\hline Test & $10.9 \pm 3.45$ & $6.1 \pm 3.55$ & $4.8 \pm 1.94$ \\
\hline Control & $9.7 \pm 3.59$ & $7.9 \pm 3.55$ & $1.8 \pm 0.81$ \\
\hline P value & 0.347 & 0.186 & $<0.001$ \\
\hline
\end{tabular}

adjunctive use of sodium bicarbonate to scaling and root planning on the established clinical parameters and to quantify the decrease in the level of inflammation by measuring the salivary MDA levels.

Sodium bicarbonate was widely used as a dentifrice before the introduction of modern toothpastes. The merit of sodium bicarbonate products seems to lie in its safety, low cost, low abrasivity, water solubility, buffering ability, compatibility with fluoride, and antibacterial properties in high concentrations. [10] Sodium bicarbonate-containing dentifrice exhibits greater bactericidal activity against Streptococcus mutans than other dentifrice. ${ }^{[11]}$ Additionally, sodium bicarbonate due to its abrasive consistency (Relative dentin abrasivity $=7$ ) works as a mechanical cleanser of teeth and gingival tissues. Thus, the topical application of sodium bicarbonate makes the removal of plaque more effective. Thus, keeping these pertinent benefits of sodium bicarbonate in perspective, its clinical usage was assessed. However, when the baseline values were compared to the values at 3 months, there was significant improvement in the clinical parameters of disease $(p<0.001)$ representing a marked improvement with treatment rendered.

In the test group at 3 months all the parameters were reduced. The GI and MDA levels showed significant improvement from baseline $(2.2 \pm .53),(10.9 \pm 3.45)$ to 3 months $(0.98 \pm 0.46),(6.1$ \pm 3.55 ) respectively (Table 1,5$)$ in test group. The clinical studies investigated the effect of periodontal treatment on oxidative stress in the oral cavity. They have demonstrated that scaling and root planing resulted in a decrease in malondialdehyde levels in the saliva of patients with periodontitis. Thus improvement of oxidative stress in the periodontium by periodontal treatment would contribute to a reduction in circulating ROS. ${ }^{[12]}$ In the present study at 3 months, as compared to control group the adjunctive use of sodium bicarbonate may have resulted in marked decrease in MDA levels and GI index scores as compared to control group. There was reduction in plaque index scores in both the groups. The test group showed the increased reduction of $(1.75 \pm 3.84)$ compared to the control group $(1.36 \pm 0.76)$. This reduction could be attributed to the mild abrasive consistency and buffering capacity of sodium bicarbonate.

There was reduction in the probing pocket depth in the test group and control group. Our results showed additional decline in PPD in test group $(2.1 \pm 0.73)$ as compared to control group $(1.4 \pm$ 0.73 ) which indicates the adjunct use of sodium bicarbonate can aid in reducing inflammation and minimize the active periodontal pocket depth.(Table 4). Christersson et al. has suggested that sodium bicarbonate packed subgingivally enhances periodontal healing when placed in periodontal pockets in conjunction with scaling and root planning procedures. ${ }^{[13]}$ The present study also showed gain in clinical attachment levels which was statistically significant. This was in accordance with a study conducted by Takigawa $\mathrm{S}$ in which 12 month post- treatment results showed that $81 \%$ of the sites that were initially $7 \mathrm{~mm}$ or deeper showed a $2 \mathrm{~mm}$ or more gain in clinical attachment after placing adjunctive sodium bicarbonate.$^{[15]}$ 
A change in the dietary carbohydrate of an individual changes the microbial composition of supragingival plaque. The carbohydrates reduce the $\mathrm{pH}$ level and create an acidic environment thus favouring the colonization and growth of the microbiota. This leads to development of caries and periodontal disease. The creation of an alkaline environment with the use of sodium bicarbonate will break this vicious cycle as an increase in the level of salivary $\mathrm{pH}$ will prevent bacterial growth and proliferation of the microbiota and reduces its pathogenicity.

Baking soda (sodium biocarbonate) due to its alkalinity (high $\mathrm{pH}$ ) will neutralize the acids in the mouth, kill microorganisms, and remove unpleasant odours. Sodium bicarbonate tends to maintain a $\mathrm{pH}$ of 8.1 (7 is neutral) even when acids, which lower $\mathrm{pH}$, or bases, which raise $\mathrm{pH}$, are added to the solution. In other words, sodium bicarbonate raise salivary $\mathrm{pH}$ that is lower than 8 (i.e. increase saliva alkalinity). Acidic saliva furthers demineralisation, while neutral or alkaline saliva furthers remineralisation of teeth and makes them caries-resistant. This alkaline $\mathrm{pH}$ in saliva will also prevent bacterial growth and proliferation of the microbiota which have got affinity to grow in acidic environment thereby reducing the pathogenicity of microflora. Also it has an antiseptic and antibacterial activity.

Periodontal pathogens have lower MICs and MBCs than the microflora from other sites, and are killed more rapidly. It was found that the higher the concentration of bicarbonate ion, the faster the organisms is killed. The antibacterial activity is solutespecific and can be attributed to the bicarbonate ion. The precise mechanism whereby bicarbonate inhibits bacterial growth is not known, although it may be due to interference with oxidative phosphorylation. The usual explanation for the growth-inhibiting effects of high salt concentrations is that hypertonic conditions create limiting water activities, whereby the cell becomes dehydrated and cellular activity stops. ${ }^{[15]}$

Significant ROS generation by neutrophils requires a minimum oxygen tension of about $1 \%$ and a $\mathrm{pH}$ of 7.0-7.5. Both these conditions are found within periodontal pockets, indicating that chronic or excess ROS production is possible at the important site of periodontal tissue damage ${ }^{[16]}$ The generalized condition of the periodontium has an effect on the MDA levels in whole saliva. Whole saliva constitute a first line of defence against free radical mediated oxidative stress. ${ }^{[17]}$ MDA that is realeased during oxidative stress becomes one constituent of saliva and can be used as a biomarker to asses the inflamatory condition. The abundance of saliva, and collection of whole saliva is easy, non-invasive and rapid and analysis of biomarkers in saliva may provide an overall assessment of disease status. ${ }^{[18,19]}$

In the present study a strong positive correlation between the degree of change in the levels of salivary MDA and the gingival index after scaling and root planing with the adjunctive use of sodium bicarbonate. Feedback information was collected from all the individuals in the test group to assess patient acceptance and perception to this unique treatment modality. The patients reported that massaging with sodium biocarbonate reduced gingival bleeding, improved gingival consistency (a feeling of gum tightening) and decrease in bad breath from the mouth.

Hence resulting in better compliance and excellent clinical outcome as sodium bicarbonate was used only as adjunct and the regular brushing methods were not disturbed.

\section{Conclusion}

The present study showed positive outcome in all the clinical parameters and significant reduction in salivary MDA levels with the adjunct use of sodium bicarbonate to non-surgical periodontal therapy. The merit of sodium bicarbonate products seems to lie in its safety, low cost, low abrasivity, water solubility, buffering ability, compatibility with fluoride, and antibacterial properties. The above properties of sodium bicarbonate have shown to improve the clinical outcomes and thus can be used as an adjunct in patients who require additional periodontal care.

\section{References}

1. Chapple IL, Matthews JB. The role of reactive oxygen and antioxidant species in periodontal tissue destruction. Periodontol 2007;43:160232.

2. Tuter G, Kurtis B, Serdar M. Interleukin-1beta and thiobarbituric acid reactive substance levels after phase I periodontal therapy in patients with chronic periodontitis. J Periodontol 2001;72:883-888.

3. Newburn E. The use of sodium bicarbonate in oral hygiene products and practice. Compend Contin Educ Dent Suppl 1997;18:02-07.

4. Drake D. Antibacterial activity of sodium bicarbonate. Compend Contin Educ Dent Suppl1997;18:17-21.

5. McCombs GB, Green ML, Root J. Effects of a chewable sodium bicarbonate oral composition on plaque and gingivitis. J Contemp Dent Pract 2001;2:31-44

6. Ohkawa $\mathrm{H}$, Ohishi N, Yagi K. Assay for lipid peroxides in animal tissues by thiobarbituric acid reaction. Anal Biochem 1979;95:351-358.

7. Satoh K. Serum Lipid peroxide in cerebrovascular disorder determined by a new colorimetric method. Clin Chim Acta 1978;90:37-43.

8. Battino M, Ferreiro MS, Quiles JL, Bompadre S, Leone L, Bullon P. Alterations in the oxidation products, antioxidant markers, antioxidant capacity and lipid patterns in plasma of patients affected by Papillon Lefevre syndrome. Free Radic Res 2003;37:603-609.

9. Akalin FA, Baltacioglu E, Alver A, Karabulut E. Lipid peroxidation levels and total oxidant status in serum, saliva and gingival crevicular fluid in patients with chronic periodontitis. J Clin Periodontol 2007;34:558565.

10. Wolff LF. Chemotherapeutic agents in the prevention and treatment of periodontal disease. Northwest Dent 1985;64:15-24.

11. Drake DR, Vargas K, Cardenzana A, Srikantha R. Enhanced bactericidal activity of Arm and Hammer Dental Care. Am J Dent 1995;8:308-312.

12. Wei D, Zhang XL, Wang YZ, Yang CX, Chen G. Lipid peroxidation levels, total oxidant status and superoxide dismutase in serum, saliva and gingival crevicular fluid in chronic periodontitis patients before and after periodontal therapy. Aust Dent J 2010;55:70-78.

13. Christersson LA, Rosling BG, Dunford RG, Wikesjö UM, Zambon JJ, Genco RJ. Monitoring of subgingival Bacteroides gingivalis and Actinobacillus actinomycetemcomitans in the management of advanced periodontitis. Adv Dent Res 1988;2:382-388. 
14. Takigawa S, Sugano N, Ochiai K, Arai N, Ota N, Ito K. Effects of sodium bicarbonate on butyric acid-induced epithelial cell damage in vitro. J Oral Sci 2008;50:413-417.

15. Newbrun E, Hoover CI, Ryder MI. Bactericidal action of bicarbonate ion on selected periodontal pathogenic microorganisms. J Periodontol 1984;55:658-667.

16. Gabig TG, Bearman SI, Babior BM. Effects of oxygen tension and $\mathrm{pH}$ on the respiratory burst of human neutrophils. Blood 1979;53:11331139 .
17. Tenovuo J, Lehtonen OP, Aaltonen AS, Vilja P, Tuohimaa P. Antimicrobial factors in whole saliva of human infants. Infect Immun 1986;51:49-53.

18. Khalili J, Biloklytska HF. Salivary malondialdehyde levels in clinically healthy and periodontal diseased individuals. Oral Diseases 2008; 14:754-760.

19. Miller CS, King CP Jr, langub MC, Kryscio RJ, Thomas MV. Salivary biomarkers of existing periodontal disease: a cross sectional study. J Am Dent Assoc 2006;137(3):322-339. 